\title{
Carcinoma Erysipelatoides Resulting from Cutaneous Metastasis of Breast Cancer
}

\author{
Hyang Hee Choi, Seung Ook Hwang, Wan Wook Kim, Hoyong Park, Soo Jung Lee', Ji Yun Jeong², \\ Jin Hyang Jung \\ Departments of Surgery, ${ }^{1}$ Oncology/Hematology, and ${ }^{2}$ Pathology, Kyungpook National University School of Medicine, Daegu, Korea
}

Cutaneous metastasis of breast cancer is easily misdiagnosed. Herein, we report two such cases. In the first case, a 49-year-old woman received neoadjuvant chemotherapy and underwent modified radical mastectomy for right locally advanced breast cancer (LABC). The erythematous skin lesion on the right chest wall was detected during ongoing trastuzumab treatment after radiotherapy. Radiation recall dermatitis was initially suspected, but the lesion persisted for 7 months, and a biopsy confirmed metastatic carcinoma showing multiple lymphatic tumor emboli. In the second case, a 42-year-old woman underwent breast-conserving surgery with axillary lymph node dissection for right $\mathrm{LABC}$, and then adjuvant chemotherapy followed by trastuzumab treatment and radiotherapy. A localized slightly pruritic erythematous patch on the right breast persisted for 2 months, and allergic dermatitis was suspected. However, a 3-cm mass developed in the right breast during trastuzumab treatment, and a biopsy confirmed a diagnosis of ductal carcinoma with multiple lymphatic tumor emboli.

Key Words: Breast, Breast neoplasms, Metastasis

\section{INTRODUCTION}

The incidence of cutaneous metastasis in patients with cancer, is estimated to be $0.6 \%$ to $10.4 \%$, and cutaneous metastasis represents $2 \%$ of all skin tumors [1]. In women, breast cancer is the most frequent primary malignancy metastasizing to the skin [2]. A large study reported that cutaneous metastasis occurs in $23.9 \%$ of patients with breast carcinoma $[2]$

Unfortunately, the diagnosis of metastatic breast cancer may be delayed because of its an uncommon clinical presentation. In addition, some therapies for breast cancer such as radiotherapy can cause skin lesions; however, many studies have emphasized the importance of awareness of the diverse manifestations of cutaneous metastasis in patients with breast cancer.

The most common clinical presentation of metastatic cutaneous breast cancer, observed in $80 \%$ of patients, is nodular metastatic breast carcinoma. Conversely, carcinoma erysipelatoides, which is character-

\section{Correspondence: Jin Hyang Jung}

Department of Surgery, Kyungpook National University Medical Center, Kyungpook National University School of Medicine, 807 Hoguk-ro, Buk-gu, Daegu 702-210, Korea

Tel: +82-53-200-2166, Fax: +82-53-200-2027, E-mail:jjh01@knu.ac.kr

Received: January 24, 2014 Accepted: April 17, 2014 ized by an erysipelas-like presentation, is only seen in $3 \%$ of patients [1], and its rare, atypical presentation often results in a delayed diagnosis.

In this small case series, we describe our experience with two patients diagnosed with carcinoma erysipelatoides on the basis of its clinical manifestations and biopsy findings.

\section{CASE REPORTS}

\section{Case 1}

In May 2010, a 49-year-old woman was diagnosed with invasive ductal carcinoma (IDC) of the right breast after an ultrasonographyguided vacuum-assisted biopsy (Mammotome ${ }^{\circledR}$; Johnson \& Johnson Health Care Systems Inc., Cincinnati, USA). Two years earlier, she had undergone total thyroidectomy for right papillary thyroid carcinoma, followed by radioiodine ablation. She was a hepatitis B virus carrier, and had no family history of breast cancer. A physical examination revealed a palpable mass in the right breast. Stage cT3N1M0 cancer was suspected, and a mass $(3.6 \mathrm{~cm})$ with right axillary metastasis was observed on ultrasonography and positron emission tomography/computed tomography (PET/CT) scans.

In December 2010, following neoadjuvant chemotherapy with four cycles of doxorubicin and cyclophosphamide (AC) and four cycles of 
paclitaxel and trastuzumab, she underwent right modified radical mastectomy (MRM), including level I and II axillary lymph node dissection (ALND) for right breast cancer. The postoperative pathological analysis revealed a grade 1 IDC $(0.8-\mathrm{cm}$ residual tumor) without axillary lymph node metastasis, stage ypT1N0M0. Immunohistochemical (IHC) profiling showed positive estrogen receptor (ER), positive progesterone receptor (PR), and positive human epidermal growth factor receptor 2 (HER2) status.

Postoperative adjuvant radiotherapy and hormone therapy with tamoxifen and trastuzumab were administered. Radiotherapy was administered in as 28 fractions (total, 5,040 cGy) to the right chest wall and five booster fractions (total, 1,000 cGy) to the site of MRM (Figure 1A). Subsequent PET/CT imaging did not reveal any recurrence or distant metastasis.

Initially, diffuse erythema with mild pain was noted at the MRM site following radiotherapy. The lesion was limited to the irradiated field, and it was prominent around the operative wound. It had almost resolved after completion of radiotherapy. However, 1 month later, during trastuzumab treatment, the erythema was gradually aggravated and was associated with mild but tolerable pain. We suspected
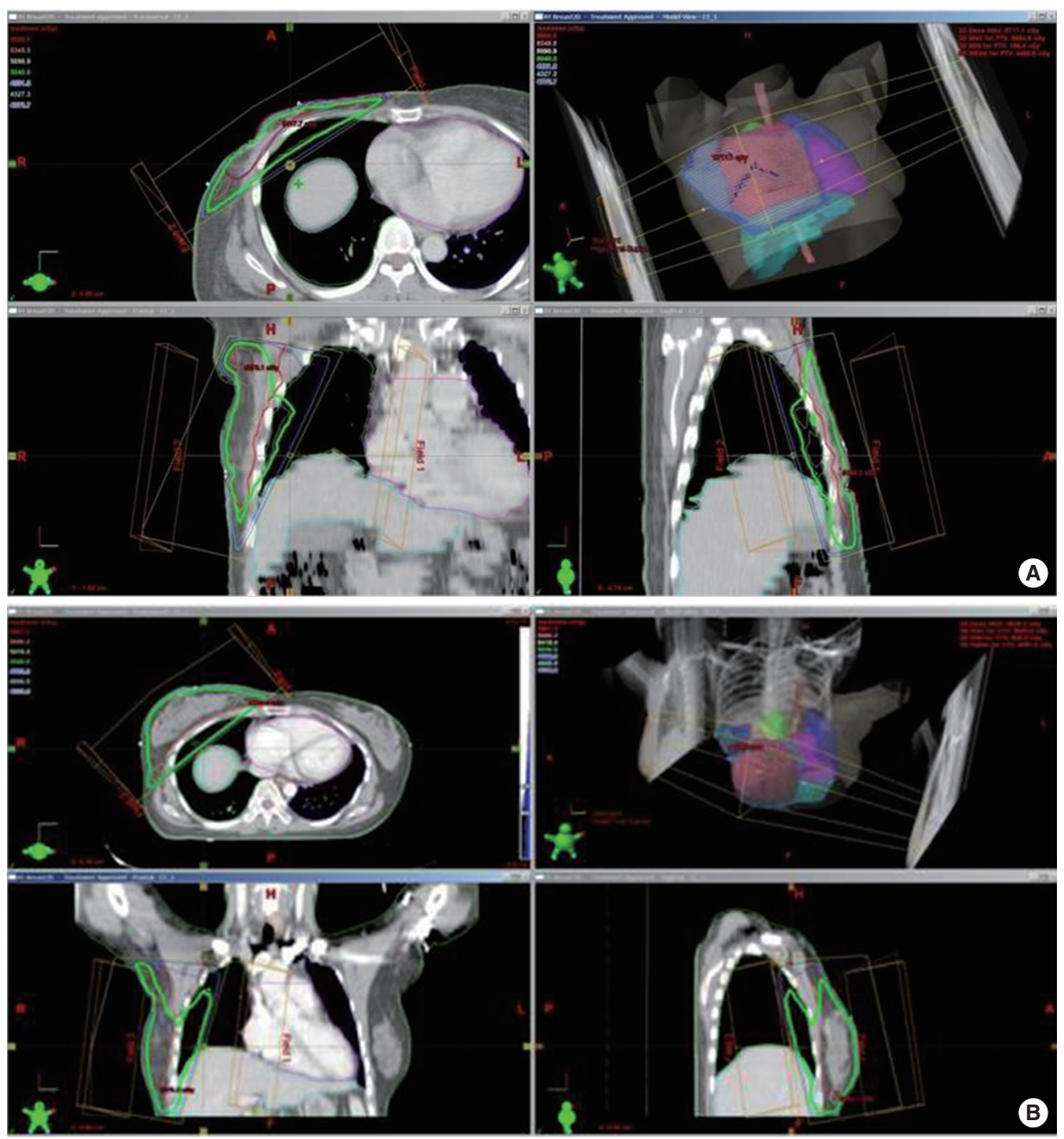

Figure 1. Postoperative adjuvant radiotherapy of case 1 and case 2. (A) Case 1. Radiotherapy performed as 28 fractions (total, 5,040 cGy) to the right chest wall and five booster fractions (total, 1,000 cGy) to the site of modified radical mastectomy (level II axillary lymph node dissection [ALND]). (B) Case 2. Radiotherapy performed as 28 fractions (total, 5,040 cGy) to the right breast and right supraclavicular nodal area and five booster fractions (total, 1,000 cGy) to the right breast tumor bed following breast-conserving surgery (ALND level II). 
trastuzumab-induced radiation recall dermatitis (RRD), particularly because the lesion corresponded to the previously irradiated field and was aggravated further during trastuzumab therapy. The lesion persisted, to differing degrees, for 7 months. Initially it was flat and fairly well demarcated, correlated with the irradiated field, and was slightly painful. As time progressed, the demarcation was still evident, but the erythema darkened and the surface was raised gradually. Eventually, the lesion displayed a slight nodular change (Figure 2A, B). Other features such as discharge, bleeding, and desquamation were not checked during the entire period. Fever was not observed; a mild but increas- ing pain persisted. A differential and confirmative diagnosis of this aggravating and enduring lesion was needed. In March 2012, a skin biopsy confirmed carcinoma erysipelatoides with metastatic cancer cells and multiple lymphatic tumor emboli obstructing the lymphatic vessels (Figure 3A, B). After this diagnosis of cutaneous metastasis, capecitabine and lapatinib treatment was initiated, and the lesion showed gradual improvement. However, in October 2012, after the 10th cycle of medication, a further skin biopsy demonstrated the persistence of metastatic carcinoma with multiple lymphatic emboli; therefore, capecitabine and lapatinib treatment was continued. Ap-
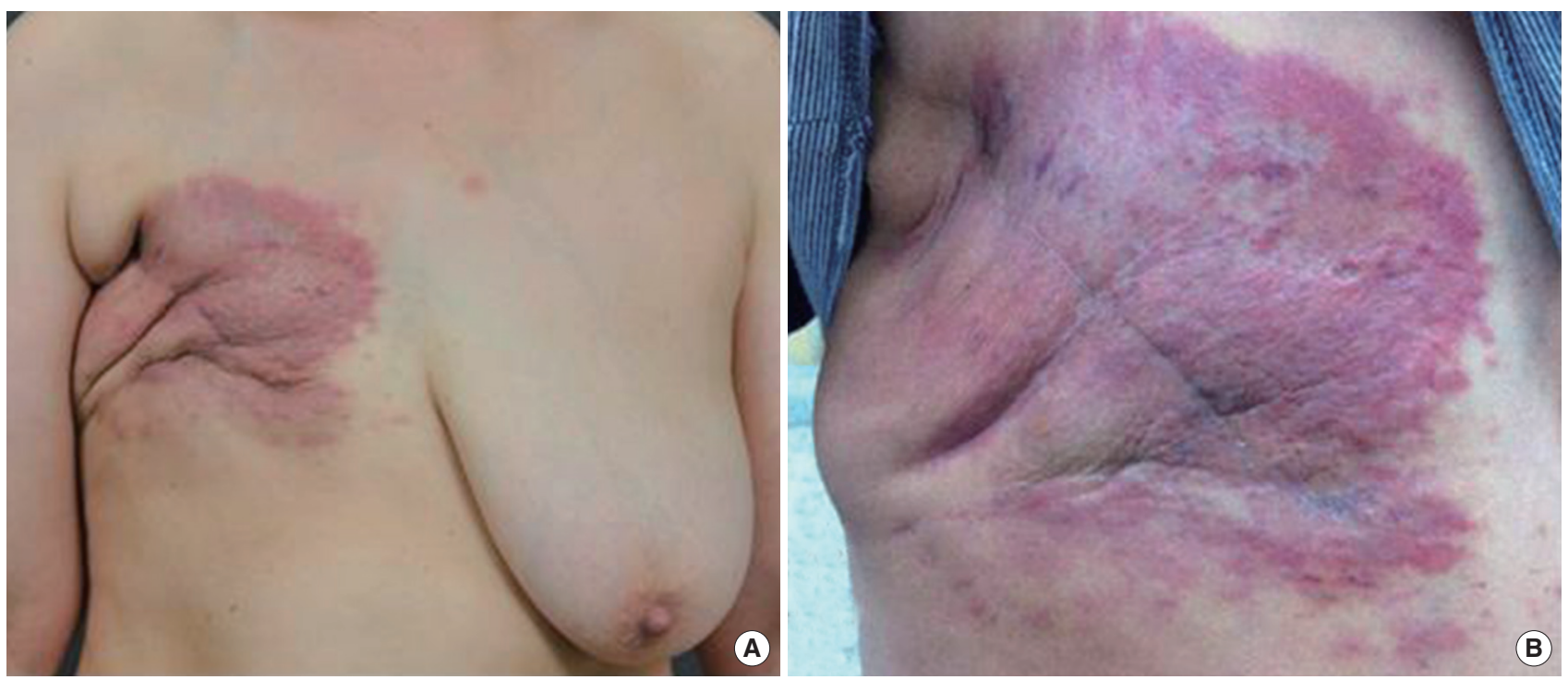

Figure 2. Features of erythematous skin lesion of case 1. (A) Initially the lesion revealed only erythema that corresponded with the previously irradiated field. The lesion was accompanied by a gradual slight nodular change. (B) Well demarcated erythema and nodular change that was aggravated around the time of skin biopsy.
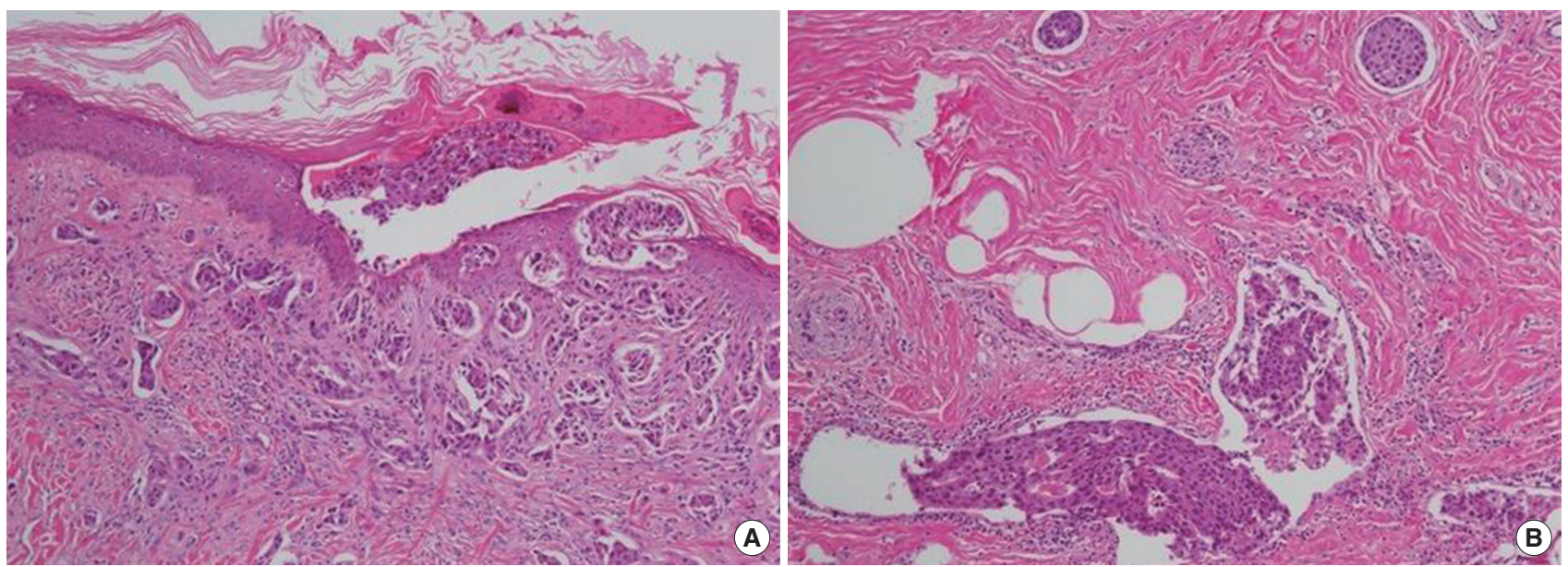

Figure 3. Microscopic findings of case 1. (A) Skin biopsy sample showed metastatic cancer cells obstructing lymphatic vessels in the form of tumor emboli (H\&E stain, $\times 100)$. (B) Skin biopsy sample showed metastatic cancer cells obstructing lymphatic vessels in the form of tumor emboli (H\&E stain, $\times 100)$. 
proximately 3 months later in January 2013, a PET/CT scan revealed metastasis in the left axillary, mediastinal, and right hilar lymph nodes from the cutaneous metastasis. The left axillary lymph node was confirmed to be a metastatic carcinoma by fine-needle aspiration cytology. The patient is currently receiving gemcitabine monotherapy.

\section{Case 2}

In December 2011, a 42-year-old woman underwent right breastconserving surgery (BCS) with level I and II ALND for right breast cancer, and immediate breast reconstruction was performed by using the rotational flap method. The postoperative pathology report revealed a 2-cm, grade 2 IDC tumor with five of 15 axillary lymph nodes, showing metastases, and the disease stage was classified as stage pT2N2M0. IHC profiling revealed a negative ER and PR status, and a positive HER2 status.

Adjuvant chemotherapy with four cycles of AC followed by four cycles of docetaxel with subsequent trastuzumab treatment was administered. In combination with trastuzumab, the patient received radiotherapy in 28 fractions (total, 5,040 cGy) to the right breast and right supraclavicular nodal area, and five booster fractions (total, 1,000 cGy) to the right breast tumor bed (Figure 1B).

In September 2012, coincident with the initiation of trastuzumab, a localized slightly pruritic erythematous skin patch developed on the right breast. The lesion was presumed to be allergic dermatitis; therefore, a $0.05 \%$ corticosteroid lotion, antihistamines, and corticosteroid medications were prescribed. The lesion gradually improved with supportive care, but persisted for 2 months. In addition, during followup, a 3-cm irregular mass was identified via breast ultrasonography and PET/CT imaging. In December 2012, a skin biopsy of the right breast skin lesion and a core needle biopsy of the right breast mass confirmed carcinoma erysipelatoides with IDC and multiple lymphatic tumor emboli. The mass was confirmed to be recurrent the breast cancer, and treatment with capecitabine and lapatinib was initiated. Currently, the skin lesion has completely disappeared, and CT scans show a reduction in the size of the breast tumor.

\section{DISCUSSION}

The diagnosis of cutaneous metastasis of breast carcinoma and the classification of its subtypes are based on clinical features and patho- logical confirmation. Although there is clinical awareness of the postsurgical occurrence of skin lesions such as erythema, nodule, and patches for many conditions, an initial diagnosis of cutaneous metastasis is rarely made because of its low incidence. In patients treated with adjuvant radiation therapy after MRM or BCS, cutaneous metastasis is easily confused with radiation dermatitis and RRD, as occurred in the first case reported here.

Radiation dermatitis can develop during the course of radiotherapy or several weeks after the completion of radiotherapy. Radiation dermatitis presents brisk erythema, desquamation, edema, discharge, bleeding by minor trauma, ulceration or necrosis and mostly accompanies pain or burning sensation. The area of lesion might irregular. The lesion might improve considerably by topical hydrocortisone or other local treatment such as dexpanthenol, calendula, and honey ointment. Antimicrobial and antioxidant properties can be helpful in wound healing.

Anticancer drugs are among the most common causes of RRD, along with radiation therapy [3]. Generally, the diagnosis of RRD is based on clinical history and appearance, and the anatomical location of the skin reaction must correlate with previously irradiated field, although sometimes it may extend beyond the irradiated area [4]. RRD includes erythema, desquamation, vesicle formation, maculopapular eruptions, edema, skin necrosis, and ulceration through the full thickness of the dermis in severe cases [4]. Commonly, RDD is recognized as an asymptomatic lesion, with no pruritus or pain.

The time interval between radiation therapy and the recall reaction varies from 2 days to 15 years, and the range of radiation doses is 10 to 61.2 Gy [4,5]. In the first case, the misdiagnosis of RRD was probably owing to the fact that the lesion corresponded with a previously irradiated field and aggravated further during trastuzumab therapy.

In cases of carcinoma erysipelatoides that are difficult to diagnose, examination of the levels of tumor markers such as cancer antigen 15-3 and carcinoembryonic antigen and the use of PET/CT imaging may not be helpful, as was clear from our experience reported here. Several days after carcinoma erysipeloides was confirmed by a bipopsy, a PET/CT scan did not show any evidence of cutaneous metastasis and tumor marker levels did not increase.

In both cases presented here, the definitive diagnosis of cutaneous metastasis was established via a skin biopsy. In erythema erysipelatoides, malignant tumor cells predominantly exist within dermal vessels. 
It is thought that lymphatic vessels are obstructed by the formation of tumor emboli in breast cancer and that this may lead to localized lymph edema, producing a peau d'orange skin appearance. Histopathologically, metastatic tumor cells are closely packed within dilated superficial and deep lymphatic vessels, and a slight perivascular infiltrate of lymphocytes and plasma cells is usually noted. IHC staining of the endothelial cells of the involved dermal vessels yields results for CD31 and podoplanin staining, suggesting that, in carcinoma erysipelatoides, tumor cell spread predominantly occurs via the lymphatic route [1]. On the other hand, the histopathologic features of RRD include a mixed inflammatory infiltrate with apoptotic and necrotic keratinocytes within the epidermis, atypical fibroblasts, dermal fibrosis, psoriasiform dermatitis, and vasodilation. IHC staining shows increased p53 expression within the epidermis after resolution of the eruption [4].

The definitive treatment modality for carcinoma erysipelatoides is not well established and the prognosis is very poor. Generally, diverse chemotherapy regimens are initiated, including capecitabine, lapatinib, gemcitabine, docetaxel, paclitaxel, epirubicin, trastuzumab, vinorelbine, and irinotecan hydrochloride [6-9]. Other treatment modalities include radiotherapy, photodynamic therapy, combination cryosurgery with hyperthermia, topical chemotherapy with $6 \%$ miltefosine solution, and surgery; however, the results have not been promising $[10]$.

A median survival of more than 80 months is extremely rare, although the range depends on factors related to the nature of the primary lesion (large tumor size and metastatic axillary lymph node involvement), treatment of the primary tumor, the inflammatory nature, and treatment of the metastatic skin lesion [10].

Tsujino et al. [10] conducted a survey of patients with carcinoma erysipelatoides corresponding to the area of postoperative radiotherapy for breast cancer. A total of 16 patients from 11 institutions were enrolled. Eight patients had BCS, and eight underwent mastectomy before radiotherapy. The patients with carcinoma erysipelatoides had several characteristics, including positive lymph node metastases, intense lymphatic invasion, and negative ER status. All patients developed subsequent distant metastases, and the prognoses were dismal despite diverse treatment attempts with chemotherapy, radiotherapy, hyperthermia, and surgery. The study reported a median survival period of 266 days for the BCS group and 1,105 days for the postmastectomy group.
In conclusion, vague and persistent skin lesions in patients with breast cancer must be carefully observed. A skin lesion biopsy should be performed as early as possible to rule out the possibility of metastasis and to prevent delays in diagnosis treatment initiation.

\section{CONFLICT OF INTEREST}

The authors declare that they have no competing interests.

\section{REFERENCES}

1. Alcaraz I, Cerroni L, Rütten A, Kutzner H, Requena L. Cutaneous metastases from internal malignancies: a clinicopathologic and immunohistochemical review. Am J Dermatopathol 2012;34:347-93.

2. Lookingbill DP, Spangler N, Helm KF. Cutaneous metastases in patients with metastatic carcinoma: a retrospective study of 4,020 patients. J Am Acad Dermatol 1993;29(2 Pt 1):228-36.

3. Shrimali RK, McPhail NJ, Correa PD, Fraser J, Rizwanullah M. Trastuzumab-induced radiation recall dermatitis: first reported case. Clin Oncol (R Coll Radiol) 2009;21:634-5.

4. Azria D, Magné N, Zouhair A, Castadot P, Culine S, Ychou M, et al. Radiation recall: a well recognized but neglected phenomenon. Cancer Treat Rev 2005;31:555-70.

5. Camidge R, Price A. Characterizing the phenomenon of radiation recall dermatitis. Radiother Oncol 2001;59:237-45.

6. Sideras K, Zahasky KM, Kaur JS. Response of cutaneous metastases from breast cancer to capecitabine. Clin Med Oncol 2008;2:415-8.

7. Chow HT, Tran K, Millar EK, Lynch J, Murrell DF. Diverse presentations of carcinoma erysipelatoides from a teaching hospital in australia. Case Rep Dermatol Med 2012;2012:134938.

8. Polat AK, Polat AV, Isik S, Alpakut S, Gungor B. Carcinoma erysipeloides: a rare form of cutaneous metastases from breast carcinoma. J Exp Clin Med 2010;27:39-41.

9. Reinders MG, Vermeer MH. Carcinoma erysipeloides in breast cancer. Surgery 2010;148:1040-1.

10. Tsujino K, Kashihara K, Kotani S, Hayakawa K, Imanaka K, Takada Y, et al. A survey of patients with inflammatory skin recurrence corresponding to the area of previous irradiation after postoperative radiotherapy for breast cancer. J Radiat Res 2011;52:797-803. 